\title{
A segment-based approach to the analysis of project evaluation problems by hesitant fuzzy sets
}

\author{
José Carlos R. Alcantud $^{1 \dagger}$, Rocío de Andrés Calle ${ }^{1 \S}$ \\ ${ }^{1}$ BORDA Research Unit and Multidisciplinary Institute of Enterprise (IME), \\ University of Salamanca, E-37007 Salamanca, Spain \\ ${ }^{\dagger}$ E-mail: jcr@usal.es \\ ${ }^{\S}$ E-mail: rocioac@usal.es
}

Received 26 October 2015

Accepted 8 January 2016

\begin{abstract}
We provide a methodology to perform an extensive and systematized analysis of problems where experts voice their opinions on the attributes of projects through a hesitant fuzzy decision matrix. This provides the decision-maker with ample information on which he or she can rely in order to make the final decision, in the form of segments instead of numbers. These segments derive from weighted average of new parametric expressions for two tenable indices of satisfaction, the distance to an ideal or the similarity to an anti-ideal, and permit to give a profuse unified picture of the relative performance of the projects. When the parameter grows, these indices tend to replicate the evaluation by respective simplistic expressions that only depend on the least, resp., the largest, evaluation and the number of evaluations in each cell.
\end{abstract}

Keywords: Hesitant fuzzy set; Group decision making; Project evaluation; Segment-based evaluation.

\section{Introduction}

The classical group decision making problem concerns the context where a group of experts have to make a decision on a set of alternatives, attending to either one or multiple criteria. The experts' opinions about the alternatives are usually characterized by their knowledge or subjective ideas, which produces a rich environment of models in order to capture the setting and reach a final decision. The literature abounds with references about the decision making process under different positions ${ }^{8,9,13,24,31,36,49,53}$.

It has long been recognized that fuzzy sets (FS) and fuzzy logic provide useful tools for the management of human subjectivity in decisionmaking contexts ${ }^{12,21,18,37}$. However in some practical problems, imprecise human knowledge (and es- pecially group knowledge) cannot be suitably represented by fuzzy sets and some generalizations are needed. This was established as early as in Zadeh $^{50}$. In this paper we are interested in a new, segment-based methodology that permits to perfom an extensive and systematized analysis of problems that are better modelled by Torra's ${ }^{38}$ hesitant fuzzy sets (HFSs, originally considered by GrattanGuinness ${ }^{16}$ ), which incorporate many-valued sets of memberships. The motivation for using this concept in decision making is clearly explained e.g., in $\mathrm{Xu}^{46}$. This reference justifies that hesitant fuzzy elements and sets have produced an extensive theoretical and applied literature $30,32,40,43,56$. Furthermore, a recent authoritative survey of HFSs is Rodríguez et $\mathrm{al}^{34}$. Here the authors summarize many useful and valuable decision making methods to solve hes- 
itant fuzzy multi-criteria decision making problems and propose further applications of HFSs to decision making.

\subsection{Our assumptions and research objectives}

We focus on the following common situation in multi-criteria decision analysis. We need to compare some alternatives or projects, and some experts evaluate their performance with respect to a set of attributes or characteristics. In this context the group knowledge on each project must be naturally represented by set-valued memberships, instead of just membership degrees as in fuzzy sets. Henceforth not only we permit imprecision or vagueness, but also a touch of uncertainty since we do not attach more value to a voiced opinion than to another one. Then the question arises: How do we analyze the problem of prioritizing these projects?

The formal statement of this question refers to hesitant fuzzy decision matrices (HFDMs), i.e., matrices whose cells contain hesitant fuzzy elements (HFEs). These HFEs collect the opinions voiced by the experts on each attribute of the succesive projects. In our description rows are associated with projects and can be assimilated with HFSs. Thus we want to compare rows in these matrices on the basis of their relative performance (as alternatives or projects).

The problem posed above, i.e., ranking HFSs or HFEs, has received attention from various authors recently. Xia and $\mathrm{Xu}^{44}$ and Farhadinia ${ }^{15}$ propose to use aggregating operators in order to associate a single HFE with each project. Then score functions give rankings of the aggregate HFEs. Xu and $\mathrm{Xia}^{47}$ rank the projects according to a direct appeal to distances. Finally, Zhou and $\mathrm{Li}^{54}$ design a lexicographic ranking that refines the $\mathrm{Xia}$ and $\mathrm{Xu}$ proposal $^{44}$. A summary of these studies related to HFSs/HFEs ranking is given in Table 1.

In order to make a broader analysis of these decision-making situations we draw inspiration from two sources. In the first place, we observe that the relative inadequacy of the projects (i.e., of their associated HFSs) can be estimated either by the 'distance' to an ideal HFS or the 'similarity' to an antiideal HFS, in the sense that the higher these eval- uations the worse the project's performance. Here we suggest respective novel parametric indicators for such proxies that incorporate the relative importance of the attributes through ex-ante allocations of weights. Their asymptotic behavior, i.e., the role of the parameter, is disclosed: when the parameter goes to infinity these indicators tend to provide an evaluation by respective simplistic expressions that only depend on the least, resp., the largest, evaluation and the number of evaluations on each attribute. In the second place, we draw inspiration from the Hurwicz approach to decision making under uncertainty ${ }^{26}$, which advocates for the combined use of 'best and worst outcomes' to assess the value of uncertain decisions. Thus the Hurwicz approach permits us to combine our two plausible parametric indices by their weighted sums, which includes both indices as extreme cases ${ }^{5}$. Their limit behavior replicates the case of the original indicators. Now for each project we obtain a segment instead of a single number, which can provide a richer analysis of the decision problem. Obviously, for any choice of the averaging aggregator a concrete ranking of projects arises.

Table 1. Summary table of studies related to ranking of HFSs or HFEs

\begin{tabular}{ll}
\hline Author(s) & Tool(s)/method(s) \\
\hline $\begin{array}{l}\text { Xia and } \mathrm{Xu}^{44} \\
\text { Farhadinia }\end{array}$ & $\begin{array}{l}\text { Aggregating operators and } \\
\text { score functions }\end{array}$ \\
\hline $\mathrm{Xu}$ and $\mathrm{Xia}^{47}$ & Distances and similarities \\
\hline Zhou and $\mathrm{Li}^{54}$ & Lexicographic procedure \\
\hline
\end{tabular}

We also report on the results of an experimental example that illustrates our proposal. In particular, we carry out a sensitivity analysis that permits to visualize the limit behaviour of our indexes in the analysis of problems characterized by HFDMs (e.g., hierarchization of projects characterized by HFSs). Finally, our approach is compared with other evaluation methods proposed by $\mathrm{Xu}^{46}$. 


\subsection{Literature review: Project evaluation problems}

Multi-criteria decision making methods (MCDM) focus on the implementation of Decision Theory in real-life problems. One of the the most complex real situations is the evaluation of projects because it includes various factors and criteria. There exist different MCDM techniques to provide solutions to this problem. The appropriateness of the method depends on the specific decision situation ${ }^{39}$. Some examples of such decision contexts are: new product development projects ${ }^{10}$, energy projects ${ }^{19}$, information technology projects ${ }^{3}$, investment projects ${ }^{2}$, etc.

Other contributions include some simple examples of different MCDM methodologies about project evaluation ${ }^{20,23,35}$. When the projects can begin on different time moments, the research on this problem is limited ${ }^{6,25,29}$.

From another point of view, Fuzzy Set Theo$r y$ has been extensively used to model uncertainty and vagueness associated with project information sources. And it has been gradually gaining importance as a tool in project selection ${ }^{4,7,17,27}$. Two milestones in this regard are Wang and Hwang ${ }^{41}$ (who develop a fuzzy integer programming model to gain an optimal investment portfolio), Chiu et al. ${ }^{11}$ and Wang et al. ${ }^{42}$ (who apply the fuzzy concept to the project selection process with the fuzzy multicriteria decision-making model (FMCDM) to select the optimal alternative).

Within the extended field of Hesitant Fuzzy Sets, which allows for cases with several degrees of membership, there are many application papers that contribute to Multicriteria Decision Making Theory $1,22,44,48,52,51,54$. Multiexpert multicriteria decision making under this requirement has been explored by Xia et al..$^{45}$.

A summary of these studies related to the evaluation problem is given in Table A.5.

\subsection{Organization of the paper}

The remaining of this paper is organized as follows. Section 2 establishes some basic definitions. Section 3 introduces our proposals for ranking hesitant fuzzy sets, as well as results concerning the asymp- totic behavior of our indices. In Section 4 we put in practice the methodology that permits to study the hierarchization of projects characterized by hesitant fuzzy sets. We visualize the asymptotic behavior of our indexes in a fully developed example, and then our results are confronted with the evaluations in existing approaches. We conclude in Section 5.

\section{Notation and definitions}

For any (possibly infinite) set $A, \mathscr{P}^{*}(A)$ denotes the set of non-empty subsets of $A$, and $\mathscr{F}^{*}(A)$ denotes the set of non-empty finite subsets of $A$.

Definition $1 .{ }^{44} \mathrm{~A}$ hesitant fuzzy element (HFE) is a non-empty, finite subset of $[0,1]$. The set of HFEs is denoted by $\mathscr{F}^{*}([0,1])$.

Henceforth we refer to $X$, a fixed set of alternatives.

Definition 2. ${ }^{38}$ A hesitant fuzzy set (HFS) on $X$ is a function from $X$ to $\mathscr{P}^{*}([0,1])$. A typical hesitant fuzzy set on $X$ is a function from $X$ to $\mathscr{F}^{*}([0,1])$. HFS $(X)$ means the set of HFSs on $X$, and the set of typical HFSs on $X$ is denoted by $\operatorname{HFS}(X)$.

Unless otherwise stated, HFSs are assumed to be typical.

Formally speaking, a (typical) HFS is a subset $M \subseteq X \times \mathscr{F}^{*}([0,1])$ such that for each $x \in X$, there is exactly one element $h_{M}(x) \in \mathscr{F}^{*}([0,1])$ such that $\left(x, h_{M}(x)\right) \in M$.

Each HFS on $X$ defines a set of membership values for each element of $X$, and in the case that the HFS is typical such set is always finite. HFEs represent the set of possible membership values of a typical hesitant fuzzy set at an alternative.

By restricting ourselves to either $\mathscr{F}^{*}([0,1])$ or $\mathscr{P}^{*}([0,1])$, i.e., non-empty HFEs, we disregard 'nonsense elements' in each HFS: on each alternative, at least one assessment must be made.

From a practical point of view, $\mathrm{Xia}$ and $\mathrm{Xu}^{44}$ show that the hesitant fuzzy set $M$ can be represented as $M=\left\{\left(x, h_{M}(x)\right) \mid x \in X\right\}$. For example, following Torra $^{38}$ we define

$$
M^{*}=\{(x, 1) \mid x \in X\}
$$

as the ideal or full HFS on $X$, and

$$
M^{-}=\{(x, 0) \mid x \in X\}
$$


as the anti-ideal or empty HFS on $X$.

Clearly, when all HFEs involved in the definition of an HFS on $X$ are singletons we can identify such HFS with a fuzzy set (FS) on $X$. That is to say, HFEs of the form

$$
M=\left\{\left(x, h_{M}(x)\right) \mid x \in X, h_{M}(x)=\left\{M_{x}\right\}\right\}
$$

can be identified with the FS on $X$ whose membership function is

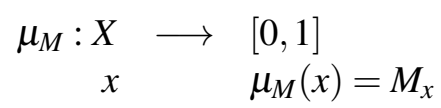

For each typical hesitant fuzzy set $M$ on $X$, we denote

$$
h_{M}(x)=\left\{h_{M}^{1}(x), \ldots, h_{M}^{l_{M}(x)}(x)\right\}
$$

where indexes are chosen so that

$$
h_{M}^{1}(x)<\ldots<h_{M}^{l_{M}(x)}(x) .
$$

In particular, the cardinality of the HFE $h_{M}(x)$ is $l_{M}(x)=\left|h_{M}(x)\right|$. Observe that if the set of membership values at an element is not finite (i.e., if we refer to a non-typical HFS) then such arrangement in increasing order cannot be made in general. In any case, because $h_{M}(x)$ is a set, repetitions are excluded by definition.

Now we proceed to formalize the general concepts of distance and similarity between HFSs.

Definition 3. [Xu and $\left.\mathrm{Xia}{ }^{47}\right] \mathrm{A}$ distance measure between HFSs on $X$ is a function $d: \operatorname{HFS}(X) \times$ $\operatorname{HFS}(X) \longrightarrow[0,1]$ that satisfies the following properties: for every $M, N \in \mathbf{H F S}(X)$,

1. $0 \leqslant d(M, N) \leqslant 1$;

2. $d(M, N)=0$ if and only if $M=N$;

3. $d(M, N)=d(N, M)$.

Definition 4. ${ }^{47} \mathrm{~A}$ similarity measure between HFSs on $X$ is a function $s: \mathbf{H F S}(X) \times \mathbf{H F S}(X) \longrightarrow[0,1]$ that satisfies the following properties: for every $M, N \in \mathbf{H F S}(X)$,
1. $0 \leqslant s(M, N) \leqslant 1$;

2. $s(M, N)=1$ if and only if $M=N$;

3. $s(M, N)=s(N, M)$.

There are similitudes between the latter concepts. When $d$ is a distance measure between HFSs on $X$, the expression $s=1-d$ defines a similarity measure between HFSs on $X$. Conversely, when $s$ is a similarity measure between HFSs on $X$, the expression $d=1-s$ defines a distance measure between HFSs on $X$. Besides $\mathrm{Xu}$ and $\mathrm{Xia}^{47}, \mathrm{Xu}^{46}$ collects many other examples of distance functions between HFSs in the literature.

\section{Ranking typical HFSs: the segment approach}

In this Section we consider the analysis of the following problem. There are $m$ alternatives or projects whose performance with regard to $n$ criteria or attributes is evaluated by a team of experts (in a range from 0 to 1). Each expert can be hesitant on the performance of the projects, therefore he or she can emit any finite number of evaluations to express his or her doubts. For each project, all evaluations by the experts on each criteria are collected into a set of values. This presumes anonymity of the experts: all opinions are equally considered in this process. Formally, this produces an HFS associated with the project: for each attribute, a finite set of values in $[0,1]$ is given. We face a problem under complete uncertainty: the importance of each particular appraisal is totally unknown.

The opinions of the experts can be captured by a hesitant fuzzy decision matrix (HFDM), i.e., an $m \times n$ matrix whose cells contain HFEs, in such way that its rows trivially define HFSs (one for each project). Columns correspond to respective evaluations of the projects by fixed criteria.

Suppose that we need to rank or prioritize the projects. The problem arises: How do we analyze the decision problem posed? 


\subsection{Analysis of the problem: the segment approach}

Several contributions have dealt with the problem posed above. $\mathrm{Xia}$ and $\mathrm{Xu}^{44}$ start by using aggregating operators in order to associate an HFE with each project, and then use a score function to rank them. Farhadinia ${ }^{15}$ proposes a variation with a different score function. $\mathrm{Xu}$ and $\mathrm{Xia}^{47}$ proceed in a more direct way: they rank the projects according to their distance to the ideal HFS. Finally, Zhou and $\mathrm{Li}^{54}$ do not produce evaluations of projects but give a lexicographic ranking that refines the proposal ${ }^{44}$.

Our proposal intends to make a richer analysis by segments instead of points: with each project we associate a segment rather than a position or a number. It has two sources of inspiration.

Firstly, we draw inspiration from the approach in $\mathrm{Xu}$ and $\mathrm{Xia}^{47}$. In order to analyze the relative performance of the projects (or of the HFSs that characterize them) we build on two relevant indicators, namely the 'distance' to the ideal HFS and the 'similarity' to the anti-ideal HFS. Both seem tenable indices of fitness for an HFS although of course, many distance and similarity indices can be used in analogy with the many proposals of distances between HFSs in the literature. In order to avoid confusions here we develop the model with a single concrete specification, namely, Definition 5 below that slightly echoes the use of the generalized hesitant weighted distance ${ }^{47}$. We leave the details of possible variations to the interested reader, i.e., specifications that replace our indicators in Definition 5 by expressions inspired on $(i)$ the generalized hesitant weighted Hausdorff distance or the generalized hybrid hesitant weighted distance ${ }^{47}$-among other distances between HFSs- or (ii) the ideas of the closedly related paper $\mathrm{Xu}$ and $\mathrm{Xia}^{48}$.

We assume that each of the attributes has associated a weight $w_{i}$ such that $w_{1}+\ldots+w_{n}=1$. Weights are indicative of the relative importance of the attributes, hence a zero weight would mean a dispensable criteria that can be omitted in the analysis. This means that we do not lose generality if we assume $w_{i}>0$ for each $i$ henceforth.
Definition 5. Given $\lambda>0$ and $\mathbf{w}=\left(w_{1}, \ldots, w_{n}\right)$ with $w_{i}>0$ for each $i$ and $w_{1}+\ldots+w_{n}=1$, the $\lambda$ adjusted hesitant weighted distance to the ideal HFS is defined as

$$
\Delta_{a h w}^{\lambda, \mathbf{w}}(M)=\sum_{i=1}^{n} \frac{w_{i}}{l_{M}\left(x_{i}\right)}\left(\sum_{j=1}^{l_{M}\left(x_{i}\right)}\left(1-h_{M}^{j}\left(x_{i}\right)\right)^{\lambda}\right)^{\frac{1}{\lambda}}
$$

for each $M \in \operatorname{HFS}(M)$ and the $\lambda$-generalized hesitant weighted similarity to the anti-ideal HFS is defined as

$$
\Sigma_{a h w}^{\lambda, \mathbf{w}}(M)=1-\sum_{i=1}^{n} \frac{w_{i}}{l_{M}\left(x_{i}\right)}\left(\sum_{j=1}^{l_{M}\left(x_{i}\right)}\left(h_{M}^{j}\left(x_{i}\right)\right)^{\lambda}\right)^{\frac{1}{\lambda}}
$$

for each $M \in \operatorname{HFS}(M)$.

Observe $\Delta_{a h w}^{\lambda, \mathbf{w}}(M)=0$ if and only if $M=M^{*}$, and $\Sigma_{a h w}^{\lambda, \mathbf{w}}(M)=0$ if and only if $M=M^{*}$. Therefore both indicators share the characteristic that the higher the evaluation of a project, the worse its performance. In the case of $\mathrm{Xia}$ and $\mathrm{Xu}^{47}$, only the analogue of the first indicator is used. In fact a direct inspection shows that when $\lambda=1$, our Definition 5 coincides with $\mathrm{Xu}$ and Xia's general hesitant weighted distance $^{47}$ between $M$ and $M^{*}$ and therefore with their hesitant weighted Hamming distance between $M$ and $M^{*}$ :

Lemma 1. If $\lambda=1$ and $\mathbf{w}=\left(w_{1}, \ldots, w_{n}\right)$ verifies $w_{i}>0$ for each $i$ and $w_{1}+\ldots+w_{n}=1$, then $\Delta_{a h w}^{\lambda, \mathbf{w}}(M)=\Sigma_{a h w}^{\lambda, \mathbf{w}}(M)$ for every $M \in \operatorname{HFS}(X)$.

Proof. For every $M \in \operatorname{HFS}(M)$,

$$
\begin{array}{r}
\Delta_{a h w}^{1, \mathbf{w}}(M)=\sum_{i=1}^{n} \frac{w_{i}}{l_{M}\left(x_{i}\right)} \sum_{j=1}^{l_{M}\left(x_{i}\right)}\left(1-h_{M}^{j}\left(x_{i}\right)\right)= \\
=\sum_{i=1}^{n} \frac{w_{i}}{l_{M}\left(x_{i}\right)}\left(l_{M}\left(x_{i}\right)-\sum_{j=1}^{l_{M}\left(x_{i}\right)} h_{M}^{j}\left(x_{i}\right)\right)= \\
=\sum_{i=1}^{n} w_{i}-\sum_{i=1}^{n}\left(\frac{w_{i}}{l_{M}\left(x_{i}\right)} \sum_{j=1}^{l_{M}\left(x_{i}\right)} h_{M}^{j}\left(x_{i}\right)\right)= \\
=1-\sum_{i=1}^{n} \frac{w_{i}}{l_{M}\left(x_{i}\right)}\left(\sum_{j=1}^{l_{M}\left(x_{i}\right)} h_{M}^{j}\left(x_{i}\right)\right)=\Sigma_{a h w}^{1, \mathbf{w}}(M)
\end{array}
$$

* Distance and similarity measures under hesitant fuzzy environment and their properties were put forward in $\mathrm{Xia}$ and $\mathrm{Xu}^{47}$ 
Secondly, we draw inspiration from the Hurwicz approach to decision making under uncertainty, which is very popular in Economics since its introduction in 1950 (cf., e.g., Luce and Raiffa ${ }^{26}$ ). In spirit it postulates the use of weighted sums of best and worst outcomes to assess the value of decisions. We can adapt it to the structure of our problem. In order to evaluate the acceptability of an HFS, both the 'distance' to the ideal HFS and the 'similarity' to the anti-ideal HFS are potentially useful. Instead of discarding one indicator in the benefit of the other, the segment approach permits us to combine both plausible indices. To be precise, in order to evaluate the hesitant fuzzy set $M$ we define a value

$$
\Lambda_{\alpha}^{\lambda, \mathbf{w}}(M)=\alpha \Delta_{a h w}^{\lambda, \mathbf{w}}(M)+(1-\alpha) \Sigma_{a h w}^{\lambda, \mathbf{w}}(M)
$$

which is a weighted sum of the distance to the ideal HFS and the similarity to the anti-ideal HFS. The weight $\alpha \in[0,1]$ can be conceived of as an index of 'enviness' because when $\alpha=1$, the indicator coincides with $\Delta_{a h w}^{\lambda, w}$, i.e., with the selected distance to the ideal HFS. When $\alpha=0$, the indicator coincides with $\Sigma_{a h w}^{\lambda, \mathbf{w}}$, i.e., with the selected similarity to the anti-ideal HFS. Intermediate values permit to use the information in both indicators, and values close to 1 , resp. 0 , bias the indicator towards $\Delta_{a h w}^{\lambda, \mathbf{w}}$, resp. $\Sigma_{a h w}^{\lambda, \mathbf{w}}$.

The higher the evaluation of an HFS by $\Lambda_{\alpha}^{\lambda, w}$, the worse its suitability. Therefore for each HFS we obtain a segment (as a function of $\alpha$ ) instead of a single number, which can provide a more extensive analysis of the decision situation to the decision-maker. Obviously, for any fixed $\alpha$ a ranking of HFSs arises, although in general this ranking is dependent on the choice of the parameter. The decision maker can observe from a single drawing for which values of the parameter a given alternative is ranked first.

Remark 1. As a consequence of Lemma 1, when $\lambda=1$ a unique ranking is obtained independently of the value of the parameter $\alpha$ because when $\mathbf{w}=\left(w_{1}, \ldots, w_{n}\right)$ verifies $w_{i}>0$ for each $i$ and $w_{1}+\ldots+w_{n}=1$, then

$$
\Lambda_{\alpha}^{1, \mathbf{w}}(M)=\Delta_{a h w}^{1, \mathbf{w}}(M)=\Sigma_{a h w}^{1, \mathbf{w}}(M)
$$

for every $M \in \operatorname{HFS}(M)$.

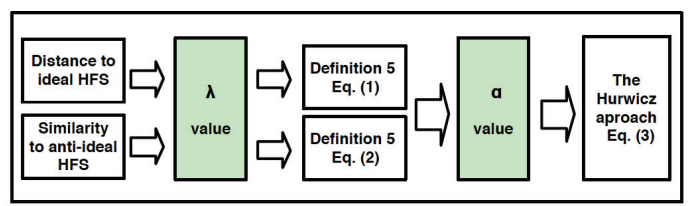

Fig. 1. The segment approach to the analysis of project evaluation problems. The use of the $\alpha$ and $\lambda$ parameters gives flexibility to our approach.

Figure 1 graphically displays the structure and the flexibility of our approach. Besides the aforementioned intuition for the $\alpha$ parameter, subsection 3.2 below intends to help us understand the role of the $\lambda$ parameter.

\subsection{Asymptotic behavior of the indicators: interpretations}

We proceed to check that using our indicators with 'large' values of the $\lambda$ parameter produces evaluations that are increasingly similar to those that derive from very simple indicators. Such indicators are crude evaluations that only rely on the number of different evaluations for each attribute and either the maximum or the minimum of such respective values. To this purpose let us define

$$
\begin{aligned}
A(M) & =\sum_{i=1}^{n} \frac{w_{i}}{l_{M}\left(x_{i}\right)} \max _{j=1, \ldots, l_{M}\left(x_{i}\right)}\left(1-h_{M}^{j}\left(x_{i}\right)\right)= \\
& =\sum_{i=1}^{n} \frac{w_{i}}{l_{M}\left(x_{i}\right)}\left(1-\min _{j=1, \ldots, l_{M}\left(x_{i}\right)} h_{M}^{j}\left(x_{i}\right)\right)
\end{aligned}
$$

for each $M \in \operatorname{HFS}(M)$ and

$$
B(M)=1-\sum_{i=1}^{n} \frac{w_{i}}{l_{M}\left(x_{i}\right)} \max _{j=1, \ldots, l_{M}\left(x_{i}\right)} h_{M}^{j}\left(x_{i}\right)
$$

for each $M \in \operatorname{HFS}(M)$. Then our claim boils down to the following statement:

Proposition 2. For every $M \in H F S(X)$,

$$
\lim _{\lambda \rightarrow \infty} \Delta_{a h w}^{\lambda, w}(M)=A(M)
$$

and also

$$
\lim _{\lambda \rightarrow \infty} \sum_{a h w}^{\lambda, w}(M)=B(M)
$$

Therefore, 


$$
\lim _{\lambda \rightarrow \infty} \Lambda_{\alpha}^{\lambda, \boldsymbol{w}}(M)=\alpha A(M)+(1-\alpha) B(M)
$$

\section{for every $M \in H F S(X)$ and $\alpha \in[0,1]$.}

Proof. We appeal to some basic properties of the $l_{p}$ norms on any $\mathbb{R}^{t}$, defined as

$$
\left\|\left(x_{1}, \cdots, x_{t}\right)\right\|_{p}=\left(\sum_{j=1}^{t}\left|x_{j}\right|^{p}\right)^{\frac{1}{p}}
$$

for every $p \geqslant 1 .^{\dagger}$

We first observe that when $M \in \operatorname{HFS}(M)$,

$\Delta_{a h w}^{\lambda, \mathbf{w}}(M)=\sum_{i=1}^{n} \frac{w_{i}}{l_{M}\left(x_{i}\right)}\left\|\left(1, \ldots l_{M}\left(x_{i}\right) \ldots, 1\right)-\left(h_{M}^{1}\left(x_{i}\right), \cdots, h_{M}^{l_{M}\left(x_{i}\right)}\left(x_{i}\right)\right)\right\|_{\lambda}$

Now it is easy to deduce the consequence $\lim _{\lambda \rightarrow \infty} \Delta_{a h w}^{\lambda, \mathbf{w}}(M)=A(M)$ : for each $i=, \ldots, n$, when $\lambda$ approaches infinity the $l_{\lambda}$ norm on $\mathbb{R}^{l_{M}\left(x_{i}\right)}$ approaches the $l_{\infty}$ or maximum norm defined as ${ }^{14}$

$$
\left\|\left(x_{1}, \cdots, x_{t}\right)\right\|_{\infty}=\max \left(\left|x_{1}\right|, \cdots,\left|x_{t}\right|\right) .
$$

The proof of the second claim is almost identical to the one above. The final statement can be trivially derived from the former ones. $\square$

An intuitive interpretation is in order. $\Delta_{a h w}^{\lambda, \mathbf{w}}(M)$ refers to similarity to an ideal HFS, and a proxy of that idea is given by the worst evaluation on each attribute, which is the information from which $A(M)$ is designed. Similarly, $\Sigma_{a h w}^{\lambda, \mathbf{w}}(M)$ refers to similarity to an anti-ideal HFS, and a proxy of that idea is given by the best evaluation on each attribute, which is the information on which $B(M)$ is designed.

\section{Experimental study}

In this section we give an experimental example to illustrate our proposal for the analysis of the hierarchization of projects respectively defined by hesitant fuzzy sets (HFSs). We also carry out a sensitivity analysis of the final outcomes in order to demonstrate the adaptability of the proposed model. Finally, we compare our conclusions with the evaluation methods proposed by $\mathrm{Xu}^{46}$, which provides experimental arguments supporting our approach.

\subsection{Evaluation framework}

Our example builds on the discussion in $\mathrm{Xu}$ and $\mathrm{Xia}^{47}$ which is adapted from Kahraman and Kaya ${ }^{19}$. Accordingly, let us suppose a society which has to compare five energy projects, denoted by alternatives $A_{i}(i=1, \ldots, 5)$. Four energy experts evaluate the performance of the five alternatives with respect to four main attributes or criteria (the example only collects all of the different possible values for each alternative and each attribute)

- $P_{1}$ : Technological. In this criterion aspects like technical feasibility, technical risk, access to technology by local agents, maturity of projects, readiness of the local agents to implement the project, multiplicative effects on the local technology basis are taken into account.

- $P_{2}$ : Environmental. Based on the project environmental impact.

- $P_{3}$ : Socio-political. Included features like the consistency of the project with the society energy policy objectives, the political acceptance of the project, the social acceptance of the project, the scope of the project vs needs to be satisfied-urgency, the appropriateness of the implementing organization, etc.

- $P_{4}$ : Economic. Estimated full cost of the project.

The criteria significance fixed by the society is $15 \%$ for technological, $30 \%$ for environmental, $20 \%$ for socio-political and $35 \%$ for economic. Consequently the attribute weight vector used along the example is $\mathbf{w}=(0.15,0.3,0.2,0.35)$.

The evaluations of the experts on the energy projects, which are based on the aforementioned criteria, are contained in a HFDM (see Figure 2 and Table 2).

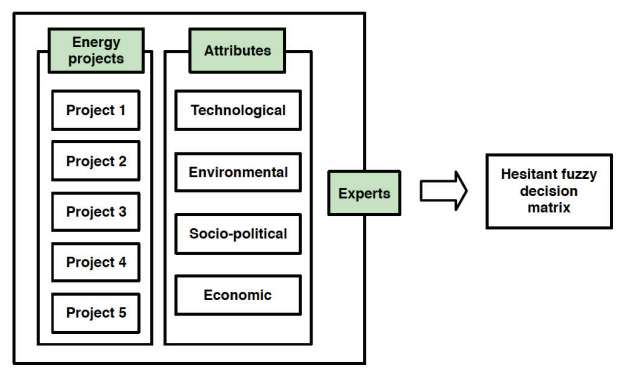

Fig. 2. Experimental study evaluation framework

\footnotetext{
$\dagger$ When $0<p<1$ such expression does not define a norm, although $\|\left(x_{1}, \cdots, x_{t}\right)||_{p}=\sum_{j=1}^{t}\left|x_{j}\right|^{p}$ does (Maddox $\left.{ }^{28}\right)$.

₹ For more details see Kahraman and Kaya ${ }^{19}$ and $\mathrm{Xu}$ and $\mathrm{Xia}^{47}$.
} 
Table 2. Hesitant fuzzy decision matrix

\begin{tabular}{lll}
\cline { 2 - 3 } & $P_{1}$ & $P_{2}$ \\
\hline$A_{1}$ & $\{0.5,0.4,0.3\}$ & $\{0.9,0.8,0.7,0.1\}$ \\
$A_{2}$ & $\{0.5,0.3\}$ & $\{0.9,0.7,0.6,0.5,0.2\}$ \\
$A_{3}$ & $\{0.7,0.6\}$ & $\{0.9,0.6\}$ \\
$A_{4}$ & $\{0.8,0.7,0.4,0.3\}$ & $\{0.7,0.4,0.2\}$ \\
$A_{5}$ & $\{0.9,0.7,0.6,0.3,0.1\}$ & $\{0.8,0.7,0.6,0.4\}$ \\
\hline & $P_{3}$ & $P_{4}$ \\
\hline$A_{1}$ & $\{0.5,0.4,0.2\}$ & $\{0.9,0.6,0.5,0.3\}$ \\
$A_{2}$ & $\{0.8,0.6,0.5,0.1\}$ & $\{0.7,0.4,0.3\}$ \\
$A_{3}$ & $\{0.7,0.5,0.3\}$ & $\{0.6,0.4\}$ \\
$A_{4}$ & $\{0.8,0.1\}$ & $\{0.9,0.8,0.6\}$ \\
$A_{5}$ & $\{0.9,0.8,0.7\}$ & $\{0.9,0.7,0.6,0.3\}$ \\
\hline
\end{tabular}

\subsection{Analysis of the hierarchization of projects: The segment approach}

In order to analyze the relative performance of the projects by means of the segment approach, we first need to produce the 'distance' to the ideal HFS and the 'similarity' to the anti-ideal HFS of each project, as measured by concrete realizations of $\lambda$ in Definition 5. To be precise, we specify the outcomes when $\lambda=1, \lambda=2$ and $\lambda=20$. Finally, we illustrate the asymptotic behavior of the indicators when $\lambda$ is large enough by comparing these outcomes with the much simpler indicators in subsection 3.2 .

- Case $\lambda=1$. Table 3 shows the results of the computations for $\Delta_{a h w}^{\lambda, \mathbf{w}}, \Sigma_{a h w}^{\lambda, \mathbf{w}}$ and $\Lambda_{\alpha}^{\lambda, \mathbf{w}}$. As proven in Lemma 1, the evaluations when $\lambda=1$ are coincident hence the conclusion $A_{5} \succ A_{3} \succ A_{4} \succ A_{1} \succ A_{2}$ irrespective of which compromise index and value of $\alpha$ we use. This consequence is shown in Figure 3 too.

Table 3. Elements for the analysis when $\lambda=1$

\begin{tabular}{ll}
\hline Alternatives & $\Delta_{a h w}^{\lambda, \mathbf{w}}=\Sigma_{a h w}^{\lambda, \mathbf{w}}=\Lambda_{\alpha}^{\lambda, \mathbf{w}}$ \\
\hline$A_{1}$ & 0.477 \\
$A_{2}$ & 0.502 \\
$A_{3}$ & 0.402 \\
$A_{4}$ & 0.429 \\
$A_{5}$ & 0.355 \\
\hline
\end{tabular}

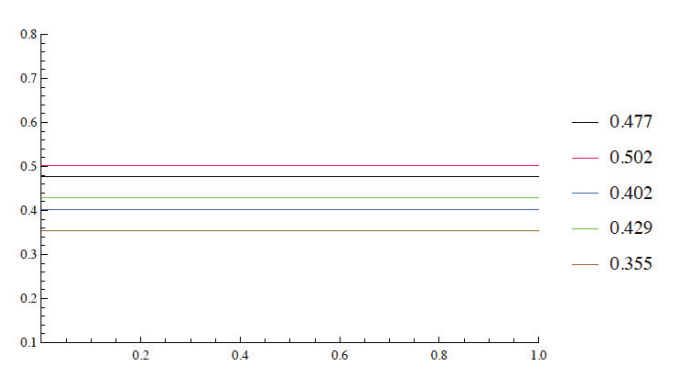

Fig. 3. A graphical display of the indicators $\Delta_{a h w}^{1, \mathbf{w}}=\Sigma_{a h w}^{1, \mathbf{w}}=\Lambda_{\alpha}^{1, \mathbf{w}}$
- Cases $\lambda=2, \lambda=20$. Tables 4 and 5 show the results of the respective computations for these values.

In order to compare the projects under a given choice of $\lambda$, the corresponding five segments $\Lambda_{\alpha}^{\lambda, w}$ can be drawn. This graphical analysis for the cases $\lambda=2, \lambda=20$ is performed in the respective Figures 4 and 5 .

Table 4. Elements for the analysis when $\lambda=2$

\begin{tabular}{lll}
\hline Index & $A_{1}$ & $A_{2}$ \\
\hline$\Delta_{a h w}^{\lambda, \mathbf{w}}$ & 0.283 & 0.298 \\
$\Sigma_{a, \mathbf{w}}^{\lambda, w}$ & 0.707 & 0.715 \\
$\Lambda_{\alpha}^{\lambda, \mathbf{w}}$ & $0.707-\alpha 0.424$ & $0.715-\alpha 0.417$ \\
\hline Index & $A_{3}$ & $A_{4}$ \\
\hline$\Delta_{a h w}^{\lambda, \mathbf{w}}$ & 0.286 & 0.287 \\
$\Sigma_{a h w}^{\lambda, \mathbf{w}}$ & 0.581 & 0.635 \\
$\Lambda_{\alpha}^{\lambda, \mathbf{w}}$ & $0.581-0.295 \alpha$ & $0.635-0.348 \alpha$ \\
\hline Index & $A_{5}$ & \\
\hline$\Delta_{a h w}^{\lambda, \mathbf{w}}$ & 0.198 & \\
$\Sigma_{a, \mathbf{w}}^{\lambda, w}$ & 0.655 & \\
$\Lambda_{\alpha}^{\lambda, \mathbf{w}}$ & $0.655-0.456 \alpha$ & \\
\hline
\end{tabular}

Table 5. Elements for the analysis when $\lambda=20$

\begin{tabular}{lll}
\hline Index & $A_{1}$ & $A_{2}$ \\
\hline$\Delta_{a h w w}^{\lambda, \mathbf{w}}$ & 0.217 & 0.227 \\
$\Sigma_{a h w w}^{\lambda, \mathbf{w}}$ & 0.795 & 0.786 \\
$\Lambda_{\alpha}^{\lambda, \mathbf{w}}$ & $0.795-0.577 \alpha$ & $0.786-0.559 \alpha$ \\
\hline & & \\
Index & $A_{3}$ & $A_{4}$ \\
\hline$\Delta_{a h w w}^{\lambda, \mathbf{w}}$ & 0.241 & 0.242 \\
$\Sigma_{a h h w}^{\lambda, \mathbf{w}}$ & 0.660 & 0.714 \\
$\Lambda_{\alpha}^{\lambda, \mathbf{w}}$ & $0.660-0.419 \alpha$ & $0.714-0.471 \alpha$ \\
\hline \multicolumn{3}{l}{} \\
Index & $A_{5}$ & \\
\hline$\Delta_{a h w w}^{\lambda, \mathbf{w}}$ & 0.153 & \\
$\Sigma_{a h w w}^{\lambda, \mathbf{w}}$ & 0.773 & \\
$\Lambda_{\alpha}^{\lambda, \mathbf{w}}$ & $0.773-0.620 \alpha$ & \\
\hline
\end{tabular}

- Case $\lambda \rightarrow \infty$. Table 6 shows the results of the computations for $A$ and $B$ and also the respective values of $I_{\alpha}$ as a function of $\alpha \in[0,1]$.

$$
I_{\alpha}\left(A_{i}\right)=\alpha A\left(A_{i}\right)+(1-\alpha) B\left(A_{i}\right) .
$$

These segments -one for each project- are uniquely determined by the HFDM. In Figure 6 , the $I_{\alpha}\left(A_{i}\right)$ segments are plotted. We recall that they only depend on the least and the largest evaluation and the number of evaluations in each cell. 
With respect to the asymptotic behavior it can be checked that the evaluations of the projects by the $\Delta_{a h w}^{\lambda, \mathbf{w}}$ indicator are identical to the respective evaluations by $A$ when $\lambda=55$, and the evaluations of the projects by the $\Sigma_{a h w}^{\lambda, \mathbf{w}}$ indicator are identical to the respective evaluations by $B$ when $\lambda=75$ (with a $10^{-6}$ precision).

Table 6. Limit values of the indicators. $I_{\alpha}$ denotes $\alpha A+(1-\alpha) B$.

\begin{tabular}{lll}
\hline Index & $A_{1}$ & $A_{2}$ \\
\hline$A$ & 0.217 & 0.227 \\
$B$ & 0.795 & 0.786 \\
$I_{\alpha}$ & $0.795-0.578 \alpha$ & $0.786-0.559 \alpha$ \\
\hline Index & $A_{3}$ & $A_{4}$ \\
\hline$A$ & 0.241 & 0.242 \\
$B$ & 0.660 & 0.715 \\
$I_{\alpha}$ & $0.660-0.419 \alpha$ & $0.715-0.472 \alpha$ \\
\hline Index & $A_{5}$ & \\
\hline$A$ & 0.15325 & \\
$B$ & 0.77425 & \\
$I_{\alpha}$ & $0.77425-\alpha 0.621$ & \\
\hline
\end{tabular}

A possible criticism to this approach is that it is fairly complex and certain factors (the $\lambda$ and $\alpha$ parameters) must be fixed. This seems to introduce ambiguity in the process of decisionmaking. Nevertheless we must point out that (i) this apparent inconvenience is common to many approaches in exactly the same setting, as subsection 4.3 below recaps; and (ii) the usual role of the analyst is to provide the decision-maker with as much information as possible, rather than making decisions. In this regard, note that our analysis provides visual information in the form of a two-dimensional graph for each choice of $\lambda$. The asymptotic behavior of these graphs (or the corresponding indexes) reveals that with only a few properly selected graphs, a complete assessment can be made.

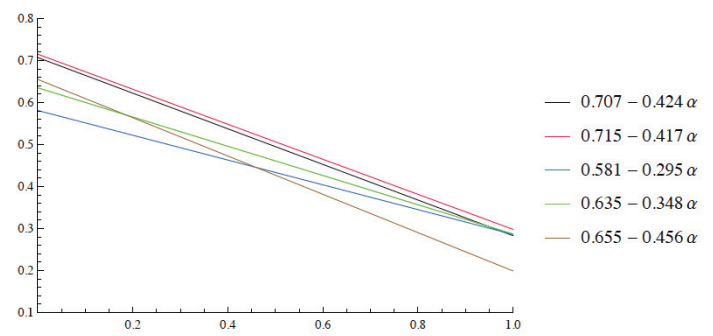

Fig. 4. A graphical display of the indicator $\Lambda_{\alpha}^{2, w}$.

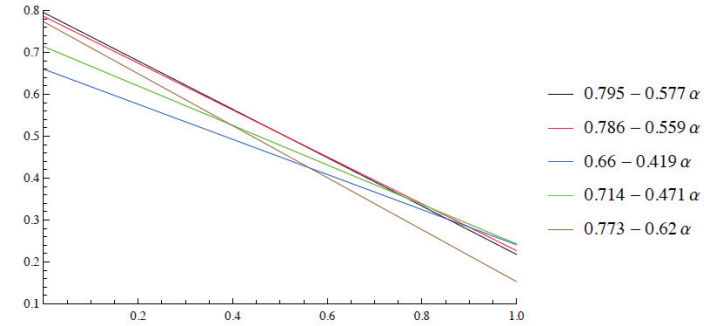

Fig. 5. A graphical display of the indicator $\Lambda_{\alpha}^{20, w}$.

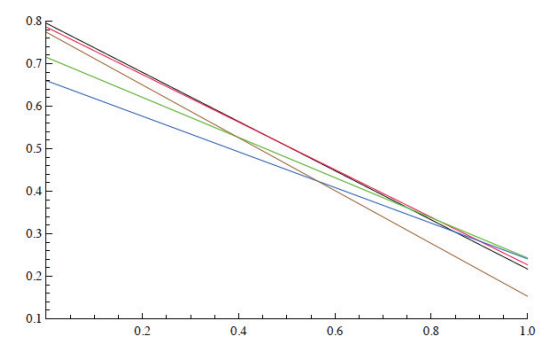

$-0.795-0.578 \alpha$

$-0.786-0.559 a$

$-0.66-0.419 \alpha$

$-0.715-0.472 \alpha$

$0.774-0.621 \alpha$

Fig. 6. A graphical display of the indicator $I_{\alpha}=\alpha A+(1-\alpha) B$.

\subsection{Discussion of the experimental study}

With the results of our experimental example set out, we now proceed to compare them with the rankings obtained from different methodologies that rank HFSs.

We begin with the procedure in $\mathrm{Xu}$ and $\mathrm{Xia}^{47}$. Table A.1 contains rankings proposed by the generalized hesitant weighted distance $\left(d_{g h w}\right)$, the generalized hesitant weighted Hausdorff distance $\left(d_{g h n h}\right)$, the generalized hybrid hesitant weighted distance $\left(d_{g h h w}\right)$ and the generalized hesitant ordered weighted distance $\left(d_{\text {ghow }}\right)$. The authors give rankings for several choices of the $\lambda$ parameter that we adopt for comparison.

In $\mathrm{Xia}$ and $\mathrm{Xu}^{44}$, Section 4 , the authors proposed to use a GHFWA $_{\lambda}$ operator (generalized hesitant fuzzy weighted averaging operator, which requires to fix a weight vector and depends on a $\lambda$ factor) in order to aggregate HFEs, and then rank the resulting HFEs according to their $\mathscr{S}_{1}$ score

$$
\mathscr{S}_{1}(h)=\frac{h^{1}+\ldots+h^{l_{h}}}{l_{h}} .
$$

Rodríguez et al. ${ }^{34}$, Section 4 , reported on many other alternative aggregators on HFEs, like GHFWG $\lambda$, GHFOWA or GHFOWG $^{44}$ or QHFOWA, HFMOWA and HFMOWG ${ }^{45}$. Furthermore, Farhadinia's $\mathscr{S}_{2}$ score or any other score on HFEs can be employed as an altermative to $\mathscr{S}_{1}$. Recall that Farhadinia ${ }^{15}$ proposed to start with a monotone non-decreasing sequence $\{\delta(1), \ldots, \delta(n), \ldots\}$ of positive numbers and then use the score

$$
\mathscr{S}_{2}(h)=\frac{\delta(1) h^{1}+\ldots+\delta\left(l_{h}\right) h^{l_{h}}}{\delta(1)+\ldots+\delta\left(l_{h}\right)} .
$$

In Table A. 2 we have computed the prioritizations with the $\mathrm{GHFWG}_{\lambda}$ and GHFWA $\lambda$ aggregators, coupled with Xia and 


\section{J.C.R. Alcantud, R. de Andrés Calle / A segment-based approach}

Xu's score. In Table A.3 we have computed the prioritizations with the same aggregators, coupled with Farhadinia's score.

In contrast, and for the current values of $\lambda$, Table A. 4 shows rankings backed up by our methodology for five values of the $\alpha$ parameter.

It seems difficult to reach clear-cut conclusions from any comparison, since already the previous analyses in Tables A.1, A. 2 and A. 3 show disparities among the rankings of the projects without a precise knowledge of their expected behavior. To check these differences Figures 7 to 10 (which illustrate the conclusions of Table A.1) and then Figures 11 to 14 (which illustrate the conclusions of Tables A.2 and A.3) are helpful and indicative. However by comparing Tables A.1 and A.4 we can note the following fact that supports the use of our segment approach with an $\alpha$ parameter. Averaging the $\Delta_{\alpha}^{\lambda, \mathbf{w}}$ and $\Sigma_{\alpha}^{\lambda, \mathbf{w}}$ indexes (with $\alpha=0.5$ or similar values) gives conclusions that are coincident with $\mathrm{Xu}$ and Xia's aforementioned verdict. However using them alone (i.e., with $\alpha=0$ or $\alpha=1$ ) produces remarkable differences. ${ }^{\S}$ Therefore we conclude that averaging distances to the ideal with similarities to the anti-ideal performs better than using any of these two approaches separately due to the fact that a segment is obtained instead of a single number for each project, which provides a richer analysis of the decision problem.

Let us also stress that our graphical illustrations prove that using both the $\Delta_{\alpha}^{\lambda, \mathbf{w}}$ and $\Sigma_{\alpha}^{\lambda, \mathbf{w}}$ indexes is not redundant.

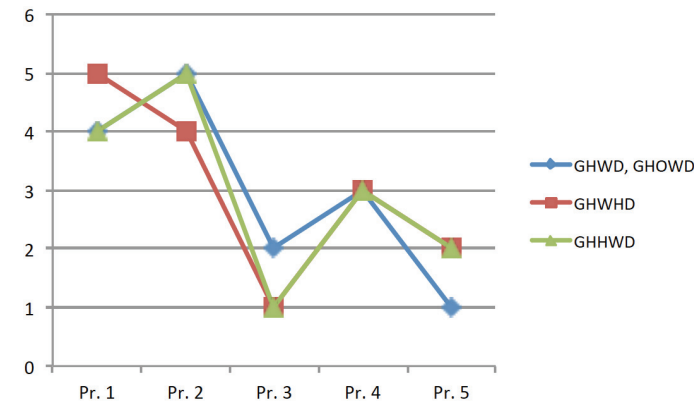

Fig. 7. A graphical display of the ranking of the five projects: application of four distances with $\lambda=1$.

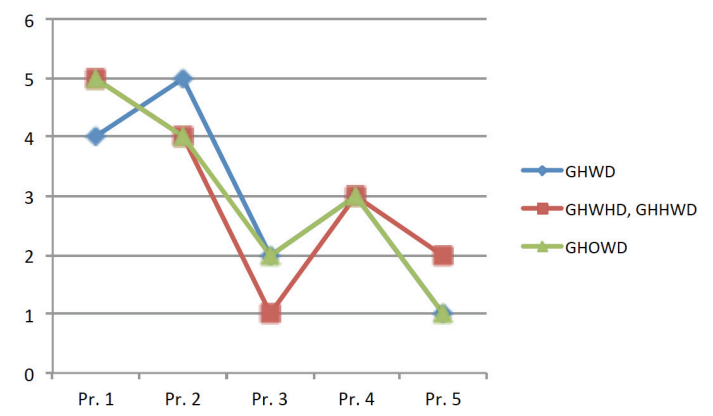

Fig. 8. A graphical display of the ranking of the five projects: application of four distances with $\lambda=2$.

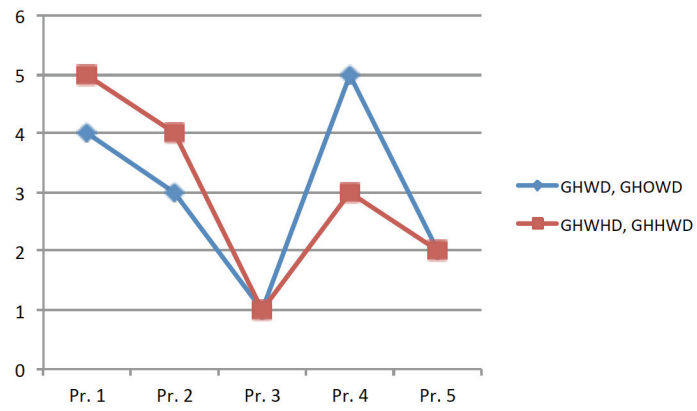

Fig. 9. A graphical display of the ranking of the five projects: application of four distances with $\lambda=6$.

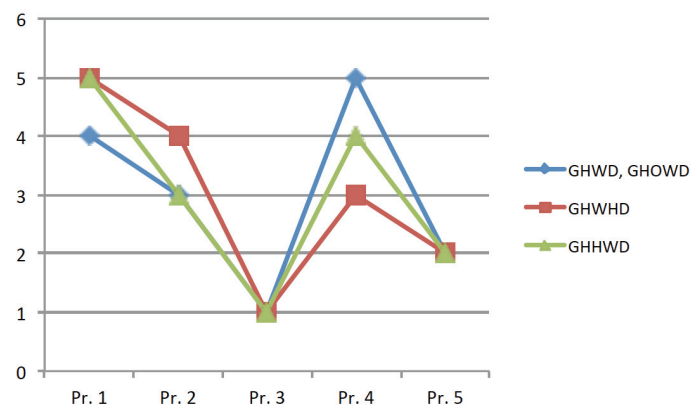

Fig. 10. A graphical display of the ranking of the five projects: application of four distances with $\lambda=10$.

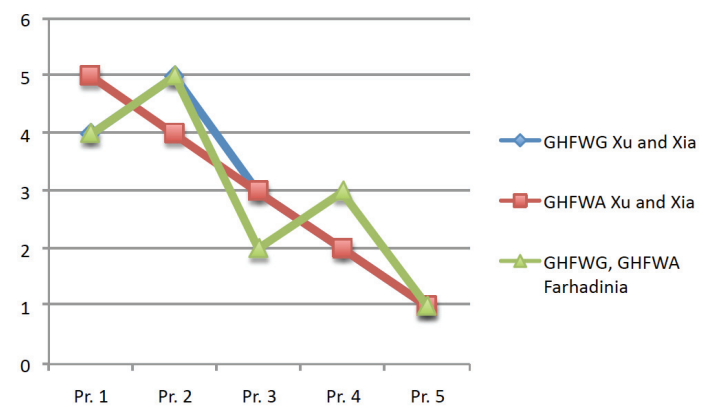

Fig. 11. A graphical display of the ranking of the five projects: application of aggregation operators with $\lambda=1$ followed by $\mathscr{S}_{1}$ and $\mathscr{S}_{2}$ scores.

$\S$ Figure 6 (see Table 6 instead) shows that the same is true when $\lambda=20$. In this case project $A_{5}$ is strictly better than $A_{3}$ which is better than the other projects, under the choice $\alpha=0.5$. This coincides with Xu and Xia's recommendation. However $A_{1}$ is better than $A_{3}$ when $\alpha=1$, and $A_{4}$ is better than $A_{5}$ when $\alpha=0$. 


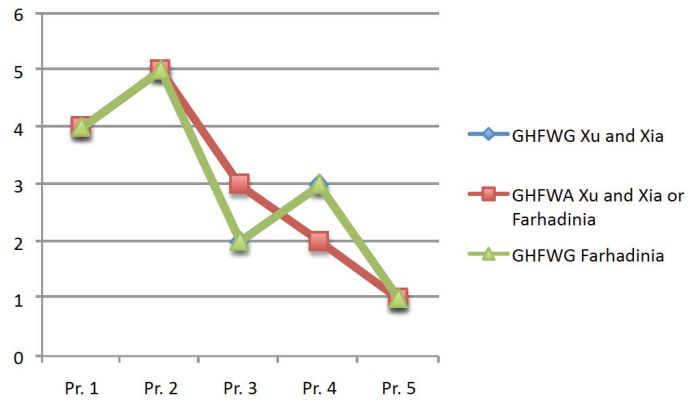

Fig. 12. A graphical display of the ranking of the five projects: application of aggregation operators with $\lambda=2$ followed by $\mathscr{S}_{1}$ and $\mathscr{S}_{2}$ scores.

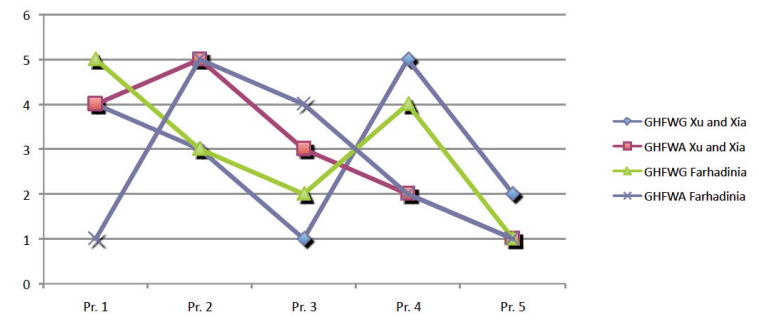

Fig. 13. A graphical display of the ranking of the five projects: application of aggregation operators with $\lambda=6$ followed by $\mathscr{S}_{1}$ and $\mathscr{S}_{2}$ scores.

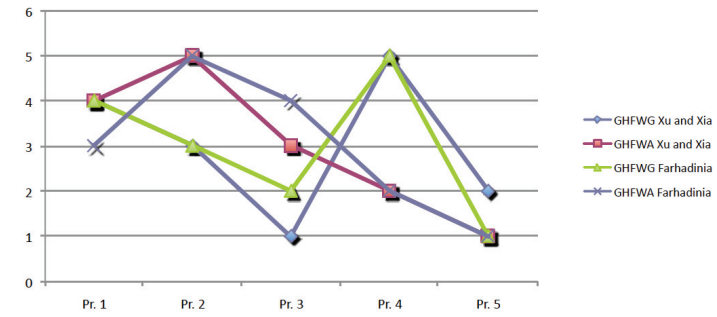

Fig. 14. A graphical display of the ranking of the five projects: application of aggregation operators with $\lambda=10$ followed by $\mathscr{S}_{1}$ and $\mathscr{S}_{2}$ scores.

\section{Conclusion}

We have provided a novel methodology that permits to perform an extensive and systematized analysis of problems with a precise specification: experts voice their opinions on the attributes of projects through a hesitant fuzzy decision matrix, that is, an $m \times n$ matrix whose cells contain HFEs. Under a specific parametric expression for two reasonable indices of satisfaction, a weighted average permits to give a profuse picture of the relative performance of the projects. A distinctive novel feature of our indicators is that the role of the parameter has been disclosed: when it grows the two indices tend to replicate the evaluation by respective simplistic expressions that only depend on the least, resp., the largest, evaluation and the number of evaluations in each cell. All these elements permit the analyst to provide the decision-maker with ample information on which he or she can rely in order to make the final decision. Moreover, an extensive graphical and numerical analysis of an example from Kahraman and Kaya ${ }^{19}$ is confronted with the corresponding analysis in $\mathrm{Xu}$ and $\mathrm{Xia}^{47}$.

With respect to related future lines of research, we already mentioned that replacing our indicators with other potentially useful expressions gives direct variations of our proposal. Particularly, the ideas in $\mathrm{Xu}$ and $\mathrm{Xia}{ }^{48}$ could be adapted to this purpose. Furthermore, the analysis of the analog problem under hesitant fuzzy linguistic information comes to mind as another natural possibility (cf., e.g., hesitant fuzzy linguistic term sets introduced by Rodríguez, Martínez and Herrera ${ }^{33}$, see also Zhu and $\mathrm{Xu}^{55}$ ).

\section{Acknowledgment}

The authors thank the Editors of the International Journal of Computational Intelligence Systems, two anonymous referees, Francisco Herrera and Luis Martínez for their valuable comments and suggestions. Financial support by the Spanish Ministerio de Ciencia e Innovación under Projects ECO2012-31933 and ECO2015-66797-P (J. C. R. Alcantud), ECO2012-32178, CGL2008-06003-C03-03/CLI and ECO2015-66797-P (R. de Andrés Calle), is gratefully acknowledged.

\section{References}

1. J. C. R. Alcantud, R. de Andrés Calle, and M. J. Torrecillas. Hesitant fuzzy worth: an innovative ranking methodology for hesitant fuzzy subsets. Applied Soft Computing, 38(1):232 - 243, 2016.

2. S. Altuntas and T. Dereli. A novel approach based on DEMATEL method and patent citation analysis for prioritizing a portfolio of investment projects. Expert Systems with Applications, 42(3):1003 - 1012, 2015.

3. A. Asosheh, S. Nalchigar, and M. Jamporazmey. Information technology project evaluation: An integrated data envelopment analysis and balanced scorecard approach. Expert Systems with Applications, 37(8):5931 - 5938, 2010.

4. G. Buyukozkan and O. Feyzioglu. A fuzzy-logicbased decision-making approach for new product development. International Journal of Production Economics, 90(1):27 - 45, 2004.

5. T. Calvo, A. Kolesárová, M. Komorníková, and R. Mesiar. Aggregation operators: Basic concepts, issues and properties. In Tomasa Calvo, Gaspar Mayor, and Radko Mesiar, editors, Aggregation Operators: New Trends and Applications, volume 97 of Studies in Fuzziness and Soft Computing, pages 3-104. Springer Berlin Heidelberg, 2002.

6. A. F. Carazo, T. Gómez, J. Molina, A. G. HernándezDíaz, F. M. Guerrero, and R. Caballero. Solving a 
comprehensive model for multiobjective project portfolio selection. Computers \& Operations Research, 37(4):630 - 639, 2010.

7. H. H. Chen, A. H. I. Lee, and Y. Tong. Prioritization and operations NPD mix in a network with strategic partners under uncertainty. Expert Systems with Applications, 33(2):337 - 346, 2007.

8. S. Chen, J. Liu, H. Wang, and J. C. Augusto. Ordering based decision making - a survey. Information Fusion, 14(4):521 - 531, 2013.

9. S. Chen, J. Liu, H. Wang, and J. C. Augusto. A group decision making model for partially ordered preference under uncertainty. Information Fusion, (forthcoming), 2015.

10. T-A. Chiang and Z. H. Che. A fuzzy robust evaluation model for selecting and ranking NPD projects using bayesian belief network and weight-restricted DEA. Expert Systems with Applications, 37(11):7408 $-7418,2010$.

11. Y-C. Chiu, B. Chen, J. Z. Shyu, and G-H. Tzeng. An evaluation model of new product launch strategy. Technovation, 26(11):1244 - 1252, 2006.

12. T. Da and Y. Xu. Evaluation on functions of urban waterfront redevelopment based on proportional 2-tuple linguistic. International Journal of Computational Intelligence Systems, 7(4):796-808, 2014.

13. M. Espinilla, R de Andrés Calle, F. J. Martínez Mimbrera, and L. Martínez. A 360-degree performance appraisal model dealing with heterogeneous information and dependent criteria. Information Sciences, 222:459-471, 2013.

14. M. Fabala et al. Functional analysis and infinitedimensional geometry. Springer, 2001.

15. B. Farhadinia. A novel method of ranking hesitant fuzzy values for multiple attribute decision-making problems. International Journal of Intelligent Systems, 28:752-767, 2013.

16. I. Grattan-Guinness. Fuzzy membership mapped onto intervals and many-valued quantities. Mathematical Logic Quarterly, 22:149-160, 1976.

17. C-C. Huang, P-Y. Chu, and Y-H. Chiang. A fuzzy AHP application in government-sponsored R\&D project selection. Omega, 36(6):1038 - 1052, 2008.

18. J. Kacprzyk and H. Nurmi. Group decision making under fuzziness. In R. Slowinski, editor, Fuzzy sets in Decision Analysis, Operations research and Statistics, pages 103-136. Springer, 1998.

19. C. Kahraman and I. Kaya. A fuzzy multicriteria methodology for selection among energy alternatives. Expert Systems with Applications, 37:62706281, 2010.

20. C. P. Lawson, P. J. Longhurst, and P. C. Ivey. The application of a new research and development project selection model in SMEs. Technovation, 26(2):242 250, 2006.
21. C. Li and Y. Dong. Multi-attribute group decision making methods with proportional 2-tuple linguistic assessments and weights. International Journal of Computational Intelligence Systems, 7(4):758-770, 2014.

22. H. Liao and Z. Xu. Satisfaction degree based interactive decision making under hesitant fuzzy environment with incomplete weights. International Journal of Uncertainty, Fuzziness and Knowledge-Based Systems, 22(04):553-572, 2014.

23. M. J. Liberatore. An expert support system for R\&D project selection. Mathematical and Computer Modelling, 11(0):260 - 265, 1988.

24. J. Lin and Y. Jiang. Some hybrid weighted averaging operators and their application to decision making. Information Fusion, 16:18 - 28, 2014.

25. S-S. Liu and C-J. Wang. Optimizing project selection and scheduling problems with time-dependent resource constraints. Automation in Construction, 20(8):1110 - 1119, 2011

26. R.D. Luce and H. Raiffa. Games and Decisions: Introduction and Critical Survey. Dover Publications, 1989.

27. L.L. Machacha and P. Bhattacharya. A fuzzy-logicbased approach to project selection. Engineering Management, IEEE Transactions on, 47(1):65-73, Feb 2000.

28. I.J. Maddox. Elements of functional analysis. Cambridge University Press, 1970.

29. A. L. Medaglia, D. Hueth, J. C. Mendieta, and J. A. Sefair. A multiobjective model for the selection and timing of public enterprise projects. Socio-Economic Planning Sciences, 42(1):31 - 45, 2008.

30. S. C. Onar, B. Oztaysi, and C. Kahraman. Strategic decision selection using hesitant fuzzy TOPSIS and interval type-2 fuzzy AHP: A case study. International Journal of Computational Intelligence Systems, 7(5):1002-1021, 2014.

31. I. Palomares, F. J. Estrella, L. Martínez, and F. Herrera. Consensus under a fuzzy context: Taxonomy, analysis framework AFRYCA and experimental case of study. Information Fusion, 20:252 -271, 2014.

32. R. M. Rodríguez, B. Bedregal, H. Bustince, Y.C. Dong, B. Farhadinia, C. Kahraman, L. Martínez, V. Torra, Y.J. Xu, Z.S. Xu, and F. Herrera. A position and perspective analysis of hesitant fuzzy sets on information fusion in decision making. towards high quality progress. Information Fusion, 29:89 - 97, 2016.

33. R.M. Rodríguez, L. Martínez, and F. Herrera. Hesitant fuzzy linguistic term sets for decision making. IEEE Transactions on Fuzzy Systems, 20(1):109-119, 2012.

34. R.M. Rodríguez, L. Martínez, V. Torra, Z. S. Xu, and F. Herrera. Hesitant fuzzy sets: state of the art and future directions. International Journal of Intelligent 
Systems, 29:495-524, 2014

35. R. Santhanam and G. J. Kyparisis. A decision model for interdependent information system project selection. European Journal of Operational Research, 89(2):380 - 399, 1996.

36. K. Suzumura. Rational Choice, Collective Decisions and Social Welfare. Cambridge University Press, Cambridge, 1983.

37. T. Tanino. Fuzzy preference orderings in group decision making. Fuzzy Sets and Systems, 12(2):117-131, 1984.

38. V. Torra. Hesitant fuzzy sets. International Journal of Intelligent Systems, 25(6):529-539, 2010.

39. D. A. Tsamboulas. A tool for prioritizing multinational transport infrastructure investments. Transport Policy, 14(1):11-26, 2007.

40. H. Wang. Extended hesitant fuzzy linguistic term sets and their aggregation in group decision making. International Journal of Computational Intelligence Systems, 8(1):14-33, 2015.

41. J. Wang and W-L. Hwang. A fuzzy set approach for $\mathrm{R} \& \mathrm{D}$ portfolio selection using a real options valuation model. Omega, 35(3):247 - 257, 2007.

42. J-J. Wang, Y-Y. Jing, C-F. Zhang, G-H.Shi, and X-T. Zhang. A fuzzy multi-criteria decision-making model for trigeneration system. Energy Policy, 36(10):3823 $-3832,2008$.

43. C. Wei, Z. Ren, and R. M. Rodríguez. A hesitant fuzzy linguistic TODIM method based on a score function. International Journal of Computational Intelligence Systems, 8(4):701-712, 2015.

44. M. Xia and Z. Xu. Hesitant fuzzy information aggregation in decision making. International Journal of Approximate Reasoning, 52:395-407, 2011.

45. M. Xia, Z. Xu, and N. Chen. Some hesitant fuzzy aggregation operators with their application in group decision making. Group Decision and Negotiation, 22:259-279, 2013.

46. Z. Xu. Hesitant Fuzzy Sets Theory, volume 314 of Studies in Fuzziness and Soft Computing. Springer International Publishing, 2014.

47. Z. Xu and M. Xia. Distance and similarity measures for hesitant fuzzy sets. Information Sciences, 181:2128-2138, 2011.

48. Z. Xu and M. Xia. On distance and correlation measures of hesitant fuzzy information. International Journal of Intelligent Systems, 26:410-425, 2011.

49. Z. Yue. Group decision making with multi-attribute interval data. Information Fusion, 14(4):551-561, 2013.

50. L. Zadeh. Outline of a new approach to analysis of complex systems and decision processes. IEEE Transactions on Systems, Man, and Cybernetics, 3:28-44, 1973.

51. X. Zhang and Z. Xu. The TODIM analysis ap- proach based on novel measured functions under hesitant fuzzy environment. Knowledge-Based Systems, 61(0):48 - 58, 2014.

52. X. Zhang and Z. Xu. Hesitant fuzzy QUALIFLEX approach with a signed distance-based comparison method for multiple criteria decision analysis. Expert Systems with Applications, 42(2):873 - 884, 2015.

53. X. Zhang, Z. Xu, and H. Wang. Heterogeneous multiple criteria group decision making with incomplete weight information: A deviation modeling approach. Information Fusion, (forthcoming), 2015.

54. X. Zhou and Q. Li. Multiple attribute decision making based on hesitant fuzzy Einstein geometric aggregation operators. Journal of Applied Mathematics, 52:Article ID 745617, 2014.

55. B. Zhu and Z. Xu. Consistency measures for hesitant fuzzy linguistic preference relations. IEEE Transactions on Fuzzy Systems, 22(1):35-45, 2014.

56. Z.Pei and L. Yi. A note on operations of hesitant fuzzy sets. International Journal of Computational Intelligence Systems, 8(2):226-239, 2015.

\section{Appendix A}

Table A.1. Rankings obtained by the Xu and Xia's distances ${ }^{47}$

\begin{tabular}{lll}
\hline$\lambda$ & $d_{g h w}$ & $d_{g h w h}$ \\
\hline 1 & $A_{5} \succ A_{3} \succ A_{4} \succ A_{1} \succ A_{2}$ & $A_{3} \succ A_{5} \succ A_{4} \succ A_{2} \succ A_{1}$ \\
2 & $A_{5} \succ A_{3} \succ A_{4} \succ A_{1} \succ A_{2}$ & $A_{3} \succ A_{5} \succ A_{4} \succ A_{2} \succ A_{1}$ \\
6 & $A_{3} \succ A_{5} \succ A_{2} \succ A_{1} \succ A_{4}$ & $A_{3} \succ A_{5} \succ A_{4} \succ A_{2} \succ A_{1}$ \\
10 & $A_{3} \succ A_{5} \succ A_{2} \succ A_{1} \succ A_{4}$ & $A_{3} \succ A_{5} \succ A_{4} \succ A_{2} \succ A_{1}$ \\
\hline
\end{tabular}

\begin{tabular}{lll}
$\lambda$ & $d_{\text {ghhw }}$ & $d_{\text {ghow }}$ \\
\hline 1 & $A_{3} \succ A_{5} \succ A_{4} \succ A_{1} \succ A_{2}$ & $A_{5} \succ A_{3} \succ A_{4} \succ A_{1} \succ A_{2}$ \\
2 & $A_{3} \succ A_{5} \succ A_{4} \succ A_{2} \succ A_{1}$ & $A_{5} \succ A_{3} \succ A_{4} \succ A_{2} \succ A_{1}$ \\
6 & $A_{3} \succ A_{5} \succ A_{4} \succ A_{2} \succ A_{1}$ & $A_{3} \succ A_{5} \succ A_{2} \succ A_{1} \succ A_{4}$ \\
10 & $A_{3} \succ A_{5} \succ A_{2} \succ A_{4} \succ A_{1}$ & $A_{3} \succ A_{5} \succ A_{2} \succ A_{1} \succ A_{4}$ \\
\hline
\end{tabular}

Table A.2. Rankings obtained by different HFSs aggregation operators and $\mathrm{Xu}$ and Xia score 44

\begin{tabular}{lll}
\hline$\lambda$ & $G F W G_{\lambda}$ & $G F W A_{\lambda}$ \\
\hline 1 & $A_{5} \succ A_{3} \succ A_{4} \succ A_{1} \succ A_{2}$ & $A_{5} \succ A_{4} \succ A_{3} \succ A_{1} \succ A_{2}$ \\
2 & $A_{5} \succ A_{3} \succ A_{4} \succ A_{1} \succ A_{2}$ & $A_{5} \succ A_{4} \succ A_{3} \succ A_{1} \succ A_{2}$ \\
6 & $A_{3} \succ A_{5} \succ A_{2} \succ A_{1} \succ A_{4}$ & $A_{5} \succ A_{4} \succ A_{3} \succ A_{1} \succ A_{2}$ \\
10 & $A_{3} \succ A_{5} \succ A_{2} \succ A_{1} \succ A_{4}$ & $A_{5} \succ A_{4} \succ A_{3} \succ A_{1} \succ A_{2}$ \\
\hline
\end{tabular}

Table A.3. Rankings obtained by different HFSs aggregation operators and Farhadinia score ${ }^{15}$

\begin{tabular}{lll}
\hline$\lambda$ & $G F W G_{\lambda}$ & $G F W A_{\lambda}$ \\
\hline 1 & $A_{5} \succ A_{3} \succ A_{4} \succ A_{1} \succ A_{2}$ & $A_{5} \succ A_{4} \succ A_{3} \succ A_{1} \succ A_{2}$ \\
2 & $A_{5} \succ A_{3} \succ A_{4} \succ A_{1} \succ A_{2}$ & $A_{5} \succ A_{4} \succ A_{3} \succ A_{1} \succ A_{2}$ \\
6 & $A_{5} \succ A_{3} \succ A_{2} \succ A_{4} \succ A_{1}$ & $A_{5} \succ A_{4} \succ A_{1} \succ A_{3} \succ A_{2}$ \\
10 & $A_{5} \succ A_{3} \succ A_{2} \succ A_{1} \succ A_{4}$ & $A_{5} \succ A_{4} \succ A_{1} \succ A_{3} \succ A_{2}$ \\
\hline
\end{tabular}


Table A.4. Rankings obtained by the segment approach $\Lambda_{\alpha}^{\lambda, \mathbf{w}}$

\begin{tabular}{lll}
\hline$\lambda$ & $\alpha$ & $\Lambda_{\alpha}^{\lambda, w}$ \\
\hline 1 & - & $A_{5} \succ A_{3} \succ A_{4} \succ A_{1} \succ A_{2}$ \\
\hline 2 & $\alpha=0$ & $A_{3} \succ A_{4} \succ A_{5} \succ A_{1} \succ A_{2}$ \\
& $\alpha=0.25$ & $A_{3} \succ A_{5} \succ A_{4} \succ A_{1} \succ A_{2}$ \\
& $\alpha=0.5$ & $A_{5} \succ A_{3} \succ A_{4} \succ A_{1} \succ A_{2}$ \\
& $\alpha=0.75$ & $A_{5} \succ A_{3} \succ A_{4} \succ A_{1} \succ A_{2}$ \\
& $\alpha=1$ & $A_{5} \succ A_{1} \succ A_{3} \succ A_{4} \succ A_{2}$ \\
\hline 6 & $\alpha=0$ & $A_{3} \succ A_{4} \succ A_{5} \succ A_{2} \succ A_{1}$ \\
& $\alpha=0.25$ & $A_{3} \succ A_{4} \succ A_{5} \succ A_{1} \succ A_{2}$ \\
& $\alpha=0.5$ & $A_{3} \succ A_{5} \succ A_{4} \succ A_{1} \succ A_{2}$ \\
& $\alpha=0.75$ & $A_{5} \succ A_{3} \succ A_{4} \succ A_{1} \succ A_{2}$ \\
& $\alpha=1$ & $A_{5} \succ A_{1} \succ A_{2} \succ A_{3} \succ A_{4}$ \\
\hline 10 & $\alpha=0$ & $A_{3} \succ A_{4} \succ A_{5} \succ A_{2} \succ A_{1}$ \\
& $\alpha=0.25$ & $A_{3} \succ A_{4} \succ A_{5} \succ A_{2} \succ A_{1}$ \\
& $\alpha=0.5$ & $A_{3} \succ A_{5} \succ A_{4} \succ A_{1} \succ A_{2}$ \\
& $\alpha=0.75$ & $A_{5} \succ A_{4} \succ A_{3} \succ A_{1} \succ A_{2}$ \\
& $\alpha=1$ & $A_{5} \succ A_{1} \succ A_{4} \succ A_{2} \succ A_{3}$ \\
\hline & &
\end{tabular}


J.C.R. Alcantud, R. de Andrés Calle / A segment-based approach

Table A.5. Summary table of studies related to evaluation projects

\begin{tabular}{|c|c|}
\hline Author(s) & Tool(s)/method(s) \\
\hline & Multi criteria decision making-based studies \\
\hline \multirow[t]{2}{*}{$\begin{array}{l}\text { Carazo et al. }{ }^{6} \text {, Lawson et al. }{ }^{20} \text {, } \\
\text { Liberatore }^{23} \text {, Liu and Wang } \\
\text { Medaglia et al. }{ }^{29} \text {, Santhanam and Kyparisis } \\
\text { Meda }^{35}\end{array}$} & $\begin{array}{l}\text { POMETHEE, TOPSIS, Data envelop analysis (DEA), } \\
\text { Analytic hierarchy process (AHP), } \\
\text { Analytic network process (ANP) }\end{array}$ \\
\hline & Fuzzy logic-based studies \\
\hline 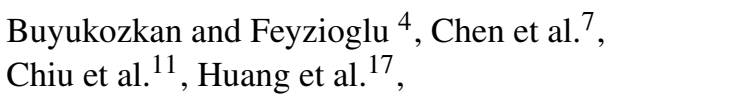 & Fuzzy POMETHEE, Fuzzy TOPSIS, \\
\hline Machacha and Bhattacharya $^{27}$, Wang and Hwang 41 & Fuzzy DEA, Fuzzy AHP, Fuzzy ANP \\
\hline $\begin{array}{l}\text { Alcantud, de Andrés Calle and Torrecillas } \\
\text { Farhadinia } \\
\text { Fa } \\
\mathrm{Xu}^{15} \text { and } \mathrm{Xia}^{48} \text {, Zhang and } \mathrm{Xu}^{22}, \mathrm{Xia} \text { and } \mathrm{Xu}^{44}, \\
51,52, \text { Zhou and } \mathrm{Li}^{54}\end{array}$ & Hesitant fuzzy logic-based studies \\
\hline
\end{tabular}

